\title{
Fragen und Antworten zur neuen Strahlenschutzverordnung
}

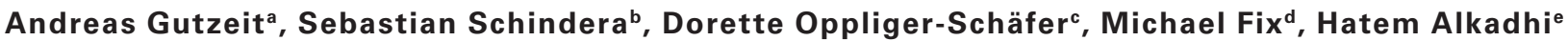

a Ressort Strahlenschutz SGR-SSR; Institut für Radiologie und Nuklearmedizin, Hirslanden Klinik St. Anna, Luzern; ETH Zürich, Departement Chemie und angewandte Biowissenschaften; ${ }^{b}$ Ressort Qualität SGR-SSR; Institut für Radiologie, Kantonsspital Aarau; ${ }^{c}$ Fachfrau Strahlenschutz Radiologische Physik SVMTRA; Klinik für Radiologie und Nuklearmedizin Universitätsspital Basel; d Präsident SGSMP; Abteilung für Medizinische Strahlenphysik, Inselspital Bern;

e Präsident der Schweizerischen Gesellschaft für Radiologie (SGR-SSR); UniversitätsSpital Zürich
\end{abstract}

Seit 2018 gilt die neue Strahlenschutzverordnung, auch die SÄZ hat darüber berichtet [1]. Diese gilt nicht nur für Radiologen, sondern auch für zahlreiche weitere Disziplinen und Fachgruppen. Wir - die Schweizerische Gesellschaft für Radiologie (SGR-SSR) - möchten über den Inhalt der Verordnung informieren und aufzeigen, welche Lösungen erarbeitet wurden.

Folgende Fragen wurden uns in den letzten Monaten häufig gestellt.

\section{Wen betreffen die neuen Gesetze?}

Antwort: Jede beruflich strahlenexponierte Person benötigt ein persönliches Dosimeter (Ärzte, MPA, MTRA, Pflege usw.). Diese Personen unterliegen der Gesetzgebung. Gemäss dem Jahresbericht Dosimetrie 2017 des BAG sind dies in der Schweiz rund 100000 Menschen [2].

\section{Wo kann man sich über die neue Gesetzgebung informieren?}

Antwort: Das Bundesamt für Gesundheit hat mehrere Artikel zur Verfügung gestellt.

- www.strahlenschutzrecht.ch

- www.legislationradioprotection.ch

- www.dirittoradioprotezione.ch

\section{Welche Fortbildungen muss ich als Arzt mit meinem Personal absolvieren (StSV Art. 175)?}

Antwort: Diesen Punkt erachten wir als aufwendig. Die Fortbildung betrifft nicht nur Radiologen, sondern alle beruflich strahlenexponierten Personen. Es besteht abhängig von der Anwendergruppe - eine Fortbildungspflicht von 4 bzw. 8 Unterrichtseinheiten à 45 Minuten. Die Fortbildungen müssen innerhalb von 5 Jahren absolviert werden. Die Art und Form der Fortbildung ist offengelassen. Frontalvorlesungen für alle ca. 100000 betroffenen Personen zu organisieren ist schwierig, teuer und nicht immer sinnvoll. Einige nutzen bereits bestehende Fortbildungen mit ihren Medizinphysikern oder Applikationsspezialisten. Für diejenigen, die weitere Lösungen benötigen, hat die SGR-SSR zwei Optionen entwickelt:

- Die SGR-SSR hat in Zusammenarbeit mit einer Firma ein E-Learning-Programm für die Schweiz auf Deutsch und Französisch entwickelt (siehe unsere Websitehttps://sgr-ssr.ch/e-learning-strahlenschutz/). Das Angebot steht allen interessierten Personen und Spitälern auch ausserhalb der SGR-SSR zur Verfügung. Die Preise für das E-Learning konnten aufgrund grosser Bestellungen deutlich gesenkt werden. SGR-SSR erhält keinerlei Provisionen; die Firma arbeitet unabhängig. Es können theoretisch alle Fortbildungen mit E-Learning durchgeführt werden; eine ergänzende praktische Anleitung ist sicher hilfreich und nützlich, aber nicht verpflichtend.

- Die SGR-SSR wird ab ihrem nächsten Jahreskongress 2020 in Fribourg gezielte Fortbildungen zum Strahlenschutz anbieten (http://www.radiologiekongress. ch). Hiermit lassen sich pro Jahr 2 Stunden Fortbildung pro Kongress absolvieren. Es sind alle herzlich eingeladen, diese Fortbildungskurse gemeinsam mit uns zu absolvieren.

\section{Was sind die wichtigsten Punkte, die alle betreffen?}

Antwort: Aus der ausführlichen Verordnung im Strahlenschutz stellen wir einige wichtige Punkte vor, die 
Prof Dr. Hatem Alkadhi Präsident der Schweizerischen Gesellschaft für Radiologie (SGR-SSR) UniversitätsSpital Zürich Institut für Diagnostische Radiologie und Interventionelle Radiologie Rämistrasse 100 CH-8091 Zürich hatem.alkadhi[at]usz.ch vor allem Ärzte und Personal ausserhalb der Radiologie betreffen

- Geteilte Rechtfertigung bildgebender Diagnostik: Es werden Zuweiserrichtlinien gefordert. Wir empfehlen Ihnen, dies mit Ihrer Radiologie abzusprechen und Standards zu verwenden, die von der KSR (Eidg. Kommission für Strahlenschutz) anerkannt werden. Der durchführende Arzt ist weiterhin verpflichtet, die Indikationsstellung des zuweisenden Kollegen zu prüfen (StSV Art. 27-30, Art. 198).

- Definition neuer Dosisbereiche in der medizinischen Bildgebung - Niedrigdosisbereich. Effektive Dosis des Patienten: $E<1 \mathrm{mSv}$; mittlerer Dosisbereich: $1 \mathrm{mSv}\langle E\langle 5 \mathrm{mSv}$; Hochdosisbereich: $E\rangle$ $5 \mathrm{mSv}$ (StSV Art. 26).

- Einbezug von Medizinphysikerinnen und -physikern auch bei Fluoroskopie z.B. im OP (neu) und im Hochdosisbereich (z.B. Kardiologie) (StSV Art. 36).

- Es gilt neu ein tieferer Dosisgrenzwert für die Augenlinse beim medizinischen Personal von 20 mSv Organ-Äquivalenzdosis pro Kalenderjahr (StSV Art. 56 Abs. 3, Art. 202 Abs. 3).

- Es besteht die Pflicht der Dosisdokumentation für alle Untersuchungen ab dem mittleren Dosisbereich. Das betrifft auch konventionelles Röntgen (mit Ausnahme von Extremitäten und Thorax-
Aufnahmen) und Mammographien. Geräte im mittleren Dosisbereich müssen mit einer Anzeige der verwendeten Dosis ausgestattet sein (RöV Art. 20 und 22).

\section{Ausblick}

Die SGR-SSR versucht, als Experte im Strahlenschutz Lösungen bei gesetzlichen Vorgaben anzubieten. Wir möchten damit unsere Kolleginnen und Kollegen aus Spitälern und Praxen unterstützen. Unser Ziel ist eine praktikable und kostenschonende Umsetzung der neuen Verordnung. Wir laden alle Interessierten ein, an unseren Initiativen teilzunehmen.

\section{Disclosure statement}

Die Autoren erklären keine Interessenverbindungen in Zusammenhang mit dem vorliegenden Manuskript.

\section{Danksagung}

Wir danken Sébastien Baechler, Philipp Trueb und dem Team des Bundesamts für Gesundheit für die inhaltliche Prüfung des Textes.

\section{Literatur}

1 Koelza HR, Linder B. Neue Strahlenschutzverordnung: Aus-, Weiter- und Fortbildung. Schweiz Ärzteztg. 2018;99:1644-6.

2 Bundesamt für Gesundheit BAG: Jahresbericht 2017 - Dosimetrie der beruflich strahlenexponierten Personen in der Schweiz; Sept. 2018.

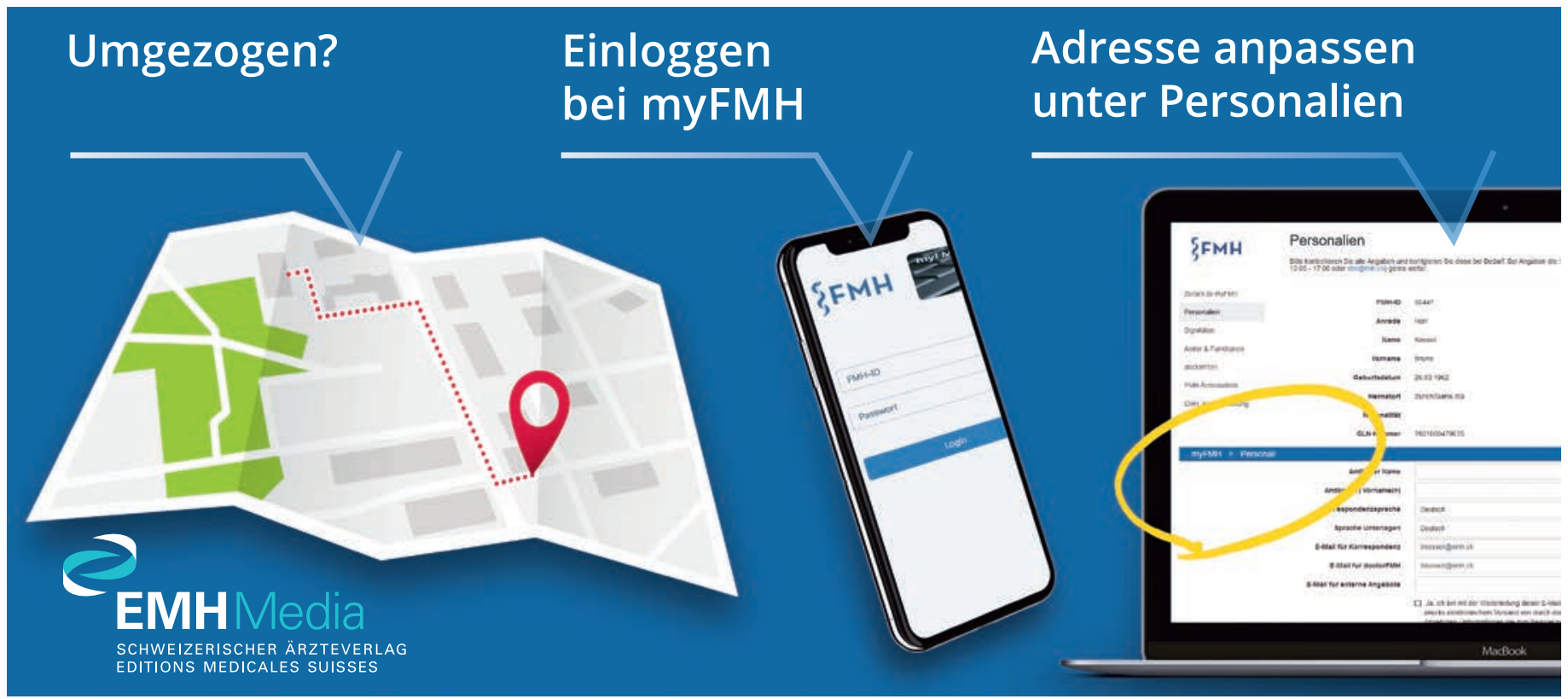

